\title{
Melting $\rho$ Meson and Thermal Dileptons
}

\author{
Ralf Rapp, Hendrik van Hees, and Trenton Strong \\ Cyclotron Institute and Physics Department, Texas A\&M University, College Station, TX 77843-3366, USA
}

Received on 4 November, 2006

\begin{abstract}
We first give a brief survey of theoretical evaluations of light vector mesons in hadronic matter, focusing on results from hadronic many-body theory. We emphasize the importance of imposing model constraints in obtaining reliable results for the in-medium spectral densities. The latter are subsequently applied to the calculation of dilepton spectra in high-energy heavy-ion collisions, with comparisons to recent NA60 data at the CERN-SPS. We discuss aspects of space-time evolution models and the decomposition of the excess spectra into different emission sources.
\end{abstract}

Keywords: Medium modifications of hadrons; Dilepton production; Ultrarelativistic heavy-Ion collisions

\section{INTRODUCTION}

The investigation of hadron properties in strongly interacting matter plays a central role for the understanding of the QCD phase diagram. On the one hand, in-medium changes of hadronic spectral functions figure into a realistic description of the bulk properties of hadronic matter, i.e., its equation of state. On the other hand, hadronic modes (especially those connected to order parameters) can serve as monitors for approaching QCD phase transitions; in fact, hadronic correlations may even play a significant role above $T_{c}$, e.g., as quark-antiquark resonances with important consequences for transport properties [1]. Of particular interest are light vector mesons $(V=\rho, \omega, \phi)$, due to their decay channel into dileptons whose invariant-mass spectra can provide undistorted information on $V$-meson spectral functions in hot and/or dense hadronic matter. A large excess of low-mass dielectrons measured in (semi-) central $\mathrm{Pb}(158 \mathrm{AGeV})+\mathrm{Au}$ collisions at the CERN Super-Proton Synchrotron (SPS) [2] has established the presence of strong medium effects in the electromagnetic spectral function. However, no definite conclusion on the underlying mechanism of the excess could be drawn (e.g., a dropping of the $\rho$ mass or a broadening of its width) [3]. More recently, the NA60 experiment measured dimuon spectra in In(158 AGeV)+In collisions [4] with much improved statistics and mass resolution allowing for more stringent conclusions: calculations based on hadronic many-body theory predicting a strongly broadened $\rho$ spectral function [5] were essentially confirmed, while those based on a dropping-mass scenario [6] are disfavored.

To evaluate consequences of medium-modified spectral densities for experimental dilepton spectra at least two more ingredients are required: (a) a space-time evolution model of the hot and dense system in A-A reactions, which (in local thermal equilibrium) provides the thermodynamic parameters (temperature, chemical potentials) for the thermal emission rates, as well as blue shifts induced by collective expansion, and (b) nonthermal sources, such as primordial Drell-Yan annihilation, "Corona" effects or emission after thermal freezeout, which become increasingly important at larger transverse momentum $\left(q_{t}\right)$ and lower collision centralities.

In this contribution we further develop our interpretation [7] of the NA60 dilepton spectra [4], including a more complete treatment of emission sources [7] and $q_{t}$ dependencies.
We briefly review calculations of in-medium low-mass vector spectral functions in Sec. II and discuss their application to NA60 data in Sec. III, including recently published $q_{t}{ }^{-}$ dependencies. Sec. IV contains our conclusions.

\section{VECTOR MESONS IN HADRONIC MATTER}

Hadronic approaches to calculate in-medium vector-meson spectral functions are typically based on effective Lagrangians with parameters (masses, coupling constants and vertex formfactors) adjusted to empirical decay rates (both hadronic and electromagnetic) and scattering data (e.g., $\pi N \rightarrow V N$ or nuclear photoabsorption). The interaction vertices are implemented into a hadronic many-body scheme to calculate selfenergy insertions of the $\rho$-propagator in hot/dense matter,

$$
D_{V}\left(M, q ; \rho_{B}, T\right)=\left[M^{2}-m_{V}^{2}-\Sigma_{V P}-\Sigma_{V B}-\Sigma_{V M}\right]^{-1}
$$

( $T$ : temperature, $\rho_{B}$ : baryon density); $\Sigma_{V M, V B}$ accounts for direct interactions of $V$ with surrounding mesons and baryons, and $\Sigma_{V P}$ for the in-medium pseudoscalar meson cloud $(2 \pi, 3 \pi$ and $K \bar{K}$ for $V=\rho, \omega$ and $\phi$, respectively).

Due to its prime importance for low-mass dilepton emission (cf. eq. (3) below), many theoretical studies have focused on the $\rho$ meson. A typical result for its spectral function [5] is shown in Fig. 1, indicating a strong broadening with increasing $T$ and $\rho_{B}$ and little mass shift.

An important question is whether the parameters of the effective Lagrangian (e.g., the bare mass, $m_{V}$, in eq. (1)) depend

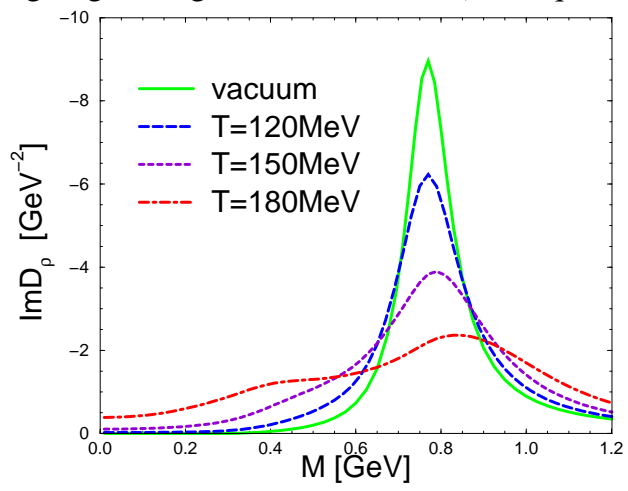

FIG. 1: $\rho$-meson spectral function, $\operatorname{Im} D_{\rho}(M, q=0)$, in hadronic matter under approximate SPS conditions $\left(\mu_{B}=330 \mathrm{MeV}\right)$. 
on $T$ and $\rho_{B}$. This requires information beyond the effectivetheory level. In Ref. [8], a Hidden-Local-Symmetry framework for introducing the $\rho$ meson into a chiral Lagrangian has been treated within a renormalization group approach at finite temperature (pion gas). While the hadronic interactions affect the in-medium $\rho$ properties only moderately, a matching of the vector and axialvector correlator to the (spacelike) operator product expansion (involving quark and gluon condensates which decrease with increasing $T$ ) requires a $T$-dependence of hadronic couplings and bare masses, inducing a dropping $\rho$-mass. On the other hand, based on QCD sum rules in cold nuclear matter (which also involve an operator product expansion), it was shown that the decrease of in-medium quark and gluon condensates can also be satisfied by an increased width of the $\rho$ spectral function [9]. The finite-density spectral functions corresponding to Fig. 1 are compatible with the QCD sum rule constraints of Ref. [9]. Since the latter are mostly driven by in-medium 4-quark condensates, $\left\langle(q \bar{q})^{2}\right\rangle$, a more accurate determination of these is mandatory to obtain more stringent conclusions on the $V$ spectral functions [10].

For the $\omega$ meson, hadronic Lagrangian calculations predict an appreciable broadening as well $\left(\Gamma_{\omega}^{\mathrm{med}} \simeq 50 \mathrm{MeV}\right.$ at normal nuclear matter density, $\rho_{0}=0.16 \mathrm{fm}^{-3}$ ), but reduced masses have also been found [11]. A recent experiment on photoproduction of $\omega$ mesons off nuclei [12] has provided evidence for a decreased $\omega$ mass, but an interpretation of the data using an $\omega$ spectral function with $90 \mathrm{MeV}$ width seems also viable [13].

Finally, $\phi$ mesons are expected to undergo significant broadening in hot and dense matter [14, 15], mostly due to modifications of its kaon cloud. Nuclear photoproduction of $\phi$ mesons [16] indicates absorption cross sections that translate into an in-medium width of $\sim 50 \mathrm{MeV}$ at $\rho_{0}$.

\section{DILEPTON SPECTRA AT CERN-SPS}

In a hot and dense medium, the equilibrium emission rate of dileptons $\left(l^{+} l^{-}\right.$with $\left.l=e, \mu\right)$ can be written as [17]

$$
\frac{d N_{l l}}{d^{4} x d^{4} q}=-\frac{\alpha^{2}}{\pi^{3}} \frac{L\left(M^{2}\right)}{M^{2}} \operatorname{Im} \Pi_{\mathrm{em}}(M, q) f^{B}\left(q_{0} ; T\right)
$$

$\left(f^{B}\right.$ : Bose distribution, $L\left(M^{2}\right)$ : lepton phase space factor). Within the vector dominance model, the low-mass regime $(M \leq 1 \mathrm{GeV})$ of the retarded e.m. current-current correlator can be saturated by the light vector mesons,

$$
\operatorname{Im} \Pi_{\mathrm{em}}=\sum_{V=\rho, \omega, \phi} \frac{m_{V}^{4}}{g_{V}^{2}} \operatorname{Im} D_{V},
$$

implying an approximate weighting of $\rho: \omega: \phi$ contributions of 10:1:2 (reflecting the values of $\Gamma_{V \rightarrow e e}$ ).

Thermal dilepton spectra in A-A collisions are obtained by convoluting the rate (2) over space-time. We employ an expanding thermal fireball whose parameters are tuned to hydrodynamic simulations (see, e.g., Ref. [18]). For central In(158 AGeV)-In, an initial Quark-Gluon Plasma (QGP) phase $\left(T_{0}=197 \mathrm{MeV}\right)$ is followed by a mixed phase and hadrochemical freezeout at $\left(\mu_{B}^{c}, T_{c}\right)=(232,175) \mathrm{MeV}$. The subsequent hadronic phase incorporates meson chemical potentials to conserve the measured particle ratios, with thermal freezeout at $T_{\mathrm{fo}} \simeq 120 \mathrm{MeV}$ after a total lifetime of $\sim 7 \mathrm{fm} / \mathrm{c}$. In the
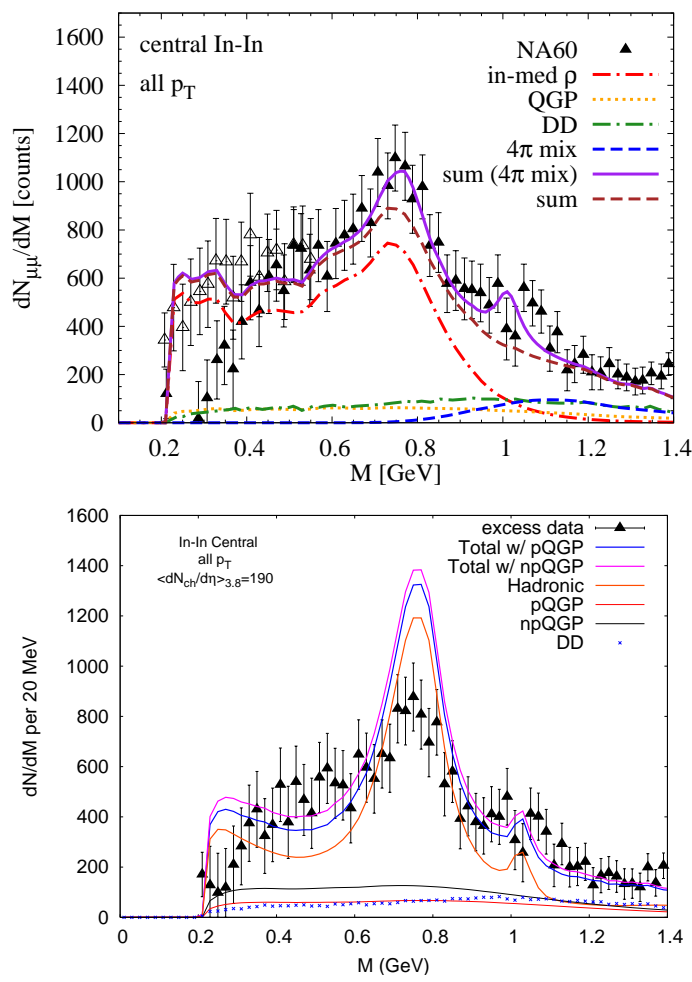

FIG. 2: Excess dilepton mass spectra in central $\operatorname{In}(158 \mathrm{AGeV})-\mathrm{In}$ compared to (a) thermal fireball calculations with thermal emission from QGP, in-medium $\rho, \omega$ and $\phi$ mesons and 4-pion-type annihilation [7] (upper panel) (b) a hydrodynamic convolution of rates from the chiral virial approach [19] (lower panel).

QGP, dilepton emission is due to $q \bar{q}$ annihilation, while in the hadron gas it is governed by in-medium $\rho, \omega$ and $\phi$ at low mass and $4 \pi$-type annihilation at $M \geq 1 \mathrm{GeV}$ (as inferred from the vacuum e.m. correlator) [7]. The calculated spectra describe the NA60 excess data well (upper panel of Fig. 2). This implies a $\rho$ spectral function that has essentially "melted" around $T_{c} \simeq 175 \mathrm{MeV}$ (while there is currently little sensitivity to inmedium $\omega$ and $\phi$ line shapes). The lower panel of Fig. 2 compares the NA60 data to thermal emission spectra based on the

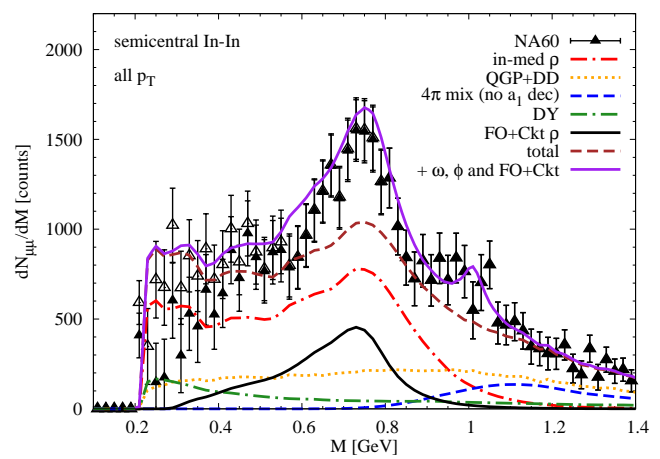

FIG. 3: Same as Fig. 2 (upper panel), but for semicentral In(158 AGeV)-In; the calculations additionally include primordial Drell-Yan annihilation and $\rho$ decays after thermal freezeout. 

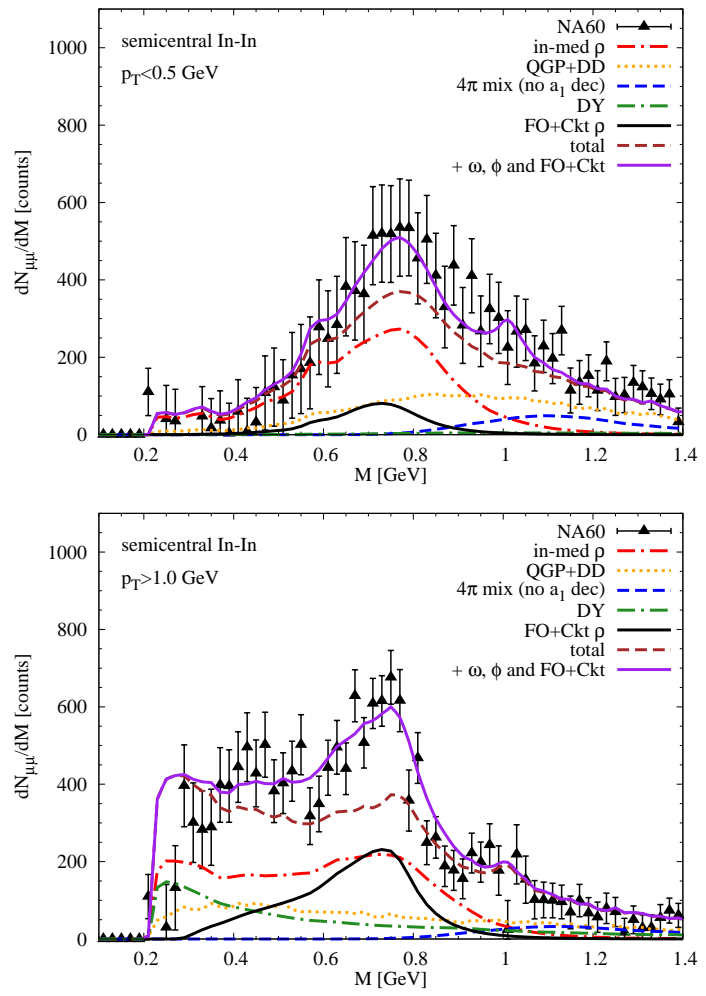

FIG. 4: Same as Fig. 3, but sliced into 2 bins of transverse pair momentum: $q_{t}<0.5 \mathrm{GeV}$ (upper panel) and $q_{t}>1.0 \mathrm{GeV}$ (lower panel).

chiral virial approach when folded over a hydrodynamic evolution [19] (the results agree well with our fireball convolution using the same input rates [20]). Albeit the virial rates imply a quenching of the $\rho$ peak, a lack of broadening is apparent. Below the free $\rho$ mass, the emission strength in the virial expansion is similar to the in-medium $\rho$ spectral function.

For a more accurate treatment of $\rho$ decays at thermal freeze- out, the rate formula (2) should be replaced by a Cooper-Frye type hydrodynamic freezeout [21] which leads to somewhat modified kinematics. Furthermore, the spectral function after freezeout is not expected to carry the full medium effects anymore. In Fig. 3 we implement these improvements, along with primordial Drell-Yan annihilation (DY) and initial "corona" $\rho$ 's, and compare to semicentral NA60 data. Since the new contributions are characterized by rather hard $q_{t}$ slopes (large "effective temperatures"), their significance primarily resides at high $q_{t}$. This is borne out when dividing the mass spectra in $q_{t}$ bins [22]: freezeout/corona $\rho$ 's and DY mostly contribute at high $q_{t}$ leading to fair agreement with experiment, cf. Fig. 4.

\section{CONCLUSIONS}

Hadronic many-body calculations predict a strong broadening, and little mass shift, of the $\rho$-meson spectral function in hot/dense hadronic matter. The good agreement with improved dilepton invariant-mass spectra by NA60 at the SPS supports the notion of a "melting" $\rho$-meson close to the expected phase boundary to the QGP. The next challenge is to establish rigorous connections to the properties of thermal QCD, especially to chiral symmetry restoration. There are promising prospects that, using chiral effective models and constraints from lattice QCD, this goal can be achieved by systematic (and quantitative) evaluations of Weinberg and QCD sum rules, which directly relate axial-/vector spectral functions to chiral order parameters.

\section{Acknowledgments}

This work has been supported by a U.S. National Science Foundation (NSF) CAREER Award under grant PHY0449489, and by the NSF REU program under grant no. PHY0354098 .
[1] M. Mannarelli and R. Rapp, Phys. Rev. C 72, 064905 (2005); H. van Hees, V. Greco, and R. Rapp, Phys. Rev. C 73, 034913 (2006).

[2] CERES/NA45 Collaboration [G. Agakichiev et al.], Eur. Phys. J. C 41, 475 (2005).

[3] R. Rapp and J. Wambach, Adv. Nucl. Phys. 25, 1 (2000).

[4] NA60 Collaboration [R. Arnaldi et al.], Phys. Rev. Lett. 96, 162302 (2006).

[5] R. Rapp, G. Chanfray, and J. Wambach, Nucl. Phys. A 617, 472 (1997).

[6] G.Q. Li, C.M. Ko, and G.E. Brown, Nucl. Phys. A 606, 568 (1996).

[7] H. van Hees and R. Rapp, Phys. Rev. Lett. 97, 102301 (2006).

[8] M. Harada and K. Yamawaki, Phys. Rept. 381, 1 (2003).

[9] S. Leupold, W. Peters, and U. Mosel, Nucl. Phys. A 628, 311 (1998).

[10] R. Thomas, S. Zschocke, and B. Kämpfer, Phys. Rev. Lett. 95, 232301 (2005).

[11] F. Klingl, T. Waas, and W. Weise, Nucl. Phys. A 650, 299
(1999).

[12] CBELSA/TAPS Collaboration [D. Trnka et al.], Phys. Rev. Lett. 94, 192303 (2005).

[13] M. Kaskulov, E. Hernandez, and E. Oset, Eur. Phys. J. A 31, 245 (2007).

[14] L. Alvarez-Ruso and V. Koch, Phys. Rev. C 65, 054901 (2002).

[15] D. Cabrera et al., Nucl. Phys. A 733, 130 (2004).

[16] T. Ishikawa et al., Phys. Lett. B 608, 215 (2005).

[17] L.D. McLerran and T. Toimela, Phys. Rev. D 31, 545 (1985).

[18] P.F. Kolb and U.W. Heinz, arXiv:nucl-th/0305084.

[19] K. Dusling, D. Teaney, and I. Zahed, Phys. Rev. C 75, 024908 (2007).

[20] H. van Hees and R. Rapp, Proceedings of the 22nd Winter Workshop on Nuclear Dynamics, EP Systema, Budapest, Hungary 2006, 91-98; and arXiv:hep-ph/0604269.

[21] F. Cooper and G. Frye, Phys. Rev. D 10, 186 (1974).

[22] S. Damjanovic [NA60 Collaboration], Eur. Phys. J. C 49, 235 (2007). 